\title{
"Honestly, this problem has affected me a lot": A qualitative exploration of the socioeconomic impacts of chronic respiratory disease among people and their communities in Sudan and Tanzania
}

Uzochukwu Egere ( $\sim$ Uzochukwu.Egere@lstmed.ac.uk)

Liverpool School of Tropical Medicine https://orcid.org/0000-0002-0282-6004

Elizabeth H Shayo

National Institute for Medical Research

Martha Chinouya

Liverpool School of Tropical Medicine

Miriam Taegtmeyer

Liverpool School of Tropical Medicine

Jane Ardery

Liverpool School of Tropical Medicine

Stella Mpagama

Kibong'oto Hospital: Kibong'oto Infectious Diseases Hospital

Nyanda Elias Ntinginya

NIMR-Mbeya Medical Research Centre

Rana Ahmed

The Epidemiological Laboratory

El Hafiz Hussein

The Epidemiological Laboratory

Asma El Sony

The Epidemiological Laboratory

Tom Wingfield

Liverpool School of Tropical Medicine Department of Clinical Sciences

Angela I N Obasi

Liverpool School of Tropical Medicine

Rachel Tolhurst

Liverpool School of Tropical Medicine 
Keywords: socioeconomic, chronic respiratory disease, stigma, healthcare seeking, psychosocial

Posted Date: October 19th, 2021

DOI: https://doi.org/10.21203/rs.3.rs-965187/v1

License: (c) (1) This work is licensed under a Creative Commons Attribution 4.0 International License. Read Full License 
"Honestly, this problem has affected me a lot": A qualitative exploration of the socioeconomic impacts of chronic respiratory disease among people and their communities in Sudan and Tanzania.

\section{Uzochukwu Egere, Elizabeth Shayo, Martha Chinouya, Miriam Taegtmeyer, Jane Ardrey, Stella} Mpagama, Nyanda Ntinginya, Rana ahmed, El Hafiz Hussein, Asma El Sony, Tom Wingfield, Angela

\section{Obasi, Rachel Tolhurst, The IMPALA Consortium}

\section{Abstract}

Background: Over 500 million people live with chronic respiratory diseases globally and approximately 4 million of these, mostly from the low- and middle-income countries including subSaharan Africa, die prematurely every year. Despite high CRD morbidity and mortality, little is known about the socioeconomic impact of CRDs in sub-Saharan Africa. We aimed to gain an in-depth understanding of the socioeconomic impact of CRDs among people with CRD to inform management of CRDs in Sudan and Tanzania.

Method: We conducted in-depth interviews with people with known or suspected CRD and focus group discussions with members of the community in Gezira state, Sudan and Dodoma region, Tanzania, to share their understanding and experience with CRD. The data was analysed using thematic framework analysis.

Results: People with CRD in both contexts reported a significantly diminished capacity to do hard physical work, resulting in both direct and indirect economic impacts for them and their families. Direct costs were incurred while seeking healthcare, including expenditures on transportation to the health facility and procurement of diagnostic tests and treatments, whilst loss of working hours and jobs resulted in substantial indirect costs. Enacted and internalised stigma leading to withdrawal and social exclusion was described by participants and resulted in part from association of chronic cough with tuberculosis and HIV/AIDS. In Sudan, asthma was described as having a negative impact on marital prospects for young women and non-disclosure related to stigma was a particular issue for young people. Impaired community participation and restrictions on social activity led to psychological stress for both people with CRD and their families.

Conclusion: Chronic respiratory diseases have substantial social and economic impacts among people with CRD and their families in Sudan and Tanzania. Stigma is particularly strong and appears to be driven in part by association of chronic cough with infectiousness. Context-appropriate measures to address economic impacts and chronic cough stigma are urgently needed as part of interventions to address chronic respiratory diseases in these sub-Saharan African contexts. 
Key words: socioeconomic, chronic respiratory disease, stigma, healthcare seeking, psychosocial

\section{Introduction}

Over five hundred million people globally live with chronic respiratory diseases (CRDs) such as asthma, chronic obstructive pulmonary disease (COPD) and bronchiectasis(1). Approximately four million of these die prematurely annually, most of whom are from low- and middle-income countries (LMICs)(2), where the estimated socioeconomic burden is also high . In sub-Saharan Africa (SSA), the increasing burden is driven by indoor air pollution due to domestic use of biomass fuel, which is used for cooking by approximately $90 \%$ of rural households(3); tobacco smoke; and post tuberculosis lung disease (4). Despite increasing recognition, CRDs are rarely prioritised by communities, health systems, or governments in SSA and robust data with which to inform CRD interventions are lacking(5). Reliable data on the prevalence of CRDs in Sub Saharan Africa (SSA) are scarce. A systematic review(6) estimated the prevalence of COPD in Sub Saharan Africa to be $13.4 \%$, which is similar to Western European and North American settings(7). Asthma incidence has been estimated to be approximately $10 \%$ in Khartoum, Sudan,(8) and recent data has shown a COPD prevalence of $16.5 \%$ in urban Sudanese adults who underwent spirometry(9). Similarly, a study in Tanzania showed an asthma prevalence of $17.6 \%$ in adolescents from urban areas(10).

CRDs are associated with severe economic burdens, including direct costs of seeking care and indirect and opportunity costs, and a negative impact on quality of life $(1,11)$. Most of the studies on CRD-associated socioeconomic burden have been conducted in high income countries whilst little is known about these impacts in LMICs despite the heavy burden of CRD morbidity and mortality. On the other hand, the negative socio-economic impacts of TB are relatively well documented. For instance, in India, female patients with TB were rejected by their families and up to $11 \%$ of children discontinued their studies with some taking up employment to support their family's finances(12). TB patients have also been reported to experience stigma and social exclusion in their communities (13) . Similarly, a systematic review of the financial burden of TB in LMICs showed that families experienced catastrophic costs, with approximately $50 \%$ of the costs incurred before treatment(14). But there is relatively little evidence relating to the socioeconomic impacts of CRDs in SSA where pathways to diagnosis and care are generally much less well defined and resourced than for TB. A cross sectional study of the socioeconomic burden of CRD in four low-income populations including Uganda, a SSA country, showed substantial disease-related productivity impairment and activity impairment among patients(15). However, this study focussed only on spirometry-diagnosed COPD and/or asthma patients. The lack of diagnostic and care pathways for CRD in most SSA countries means that there are many symptomatic people without a diagnosis or consistent care whose 
experiences may differ. More exploratory in-depth understanding of the circumstances, responses and actions of patients and their communities is required to provide a broader context for addressing CRDs within the health system in SSA.

We aimed to gain an in depth understanding of the socioeconomic impact of CRDs on patients, their families and communities, as part of the development of context-specific interventions to integrate CRD care into routine health systems in Sudan and Tanzania.

\section{Methods}

We used qualitative methods to explore the perceptions, experiences and priorities of community members and people with known or suspected chronic respiratory disease to gain an in-depth, 'emic' understanding of their interpretation and experience of CRD. We used qualitative methods because they are best suited to answer questions about experience, meaning and perspectives from the standpoint of the participant(16). We used an interpretivist approach (17) in order to allow participants to share their understanding and interpretation of CRDs and how these meanings influenced their experiences. We chose in depth interviews (IDIs) with people suspected of, or diagnosed with, CRD and focus group discussions (FGDs) with a representative group of community members. The constitution of the FGDs into same gender and age groups encouraged open discussions and allowed biases and beliefs to be freely discussed.

\section{Study sites and context}

This study was nested in the multidisciplinary "International Multidisciplinary Programme to Address Lung Health and TB in Africa (IMPALA)" consortium, which focused on generating knowledge and implementable solutions for CRDs in Sub Saharan Africa. Sudan and Tanzania were selected for the IMPALA's health systems research work package . Our baseline situation analysis in Dodoma region of Tanzania and Gezira state in Sudan explored the readiness of health facilities in these areas to deliver care for $\operatorname{CRD}(5)$. Both sites are mainly rural and semi-urban, with inhabitants engaged mostly in informal livelihoods. The sites were selected in collaboration with the local Ministries of Health because they were perceived to be exemplars of good practice that could be built on to develop CRD services. Gezira State in Sudan was selected because of an existing pilot programme offering integrated lung health services focusing on asthma standard case management (18). These services were developed by the IMPALA collaborating institution, Epidemiological Laboratory (EPILAB), a research non-governmental organization (NGO) with research infrastructure in Gezira. Dodoma Region in Tanzania was selected due to an embedded regional TB Control Program infrastructure 
and well-functioning community-based referral and follow-up system for people with chronic cough suspected to have TB even when their TB investigations are negative. The community referral system in Tanzania maintains contact with community members through designated community health workers who identify people with chronic cough who were found to be negative for TB but continue to seek care in the health facilities for on-going respiratory symptoms.

\section{Participant selection and recruitment}

For IDIs, purposive sampling was used to select participants with confirmed or suspected CRD(19). In Sudan, patients were purposively selected from the asthma standard case management programme at EPILAB sites to include men and women with varied asthma severity and from catchment communities closer and farther away from the health facility. Diagnosis of asthma was made by clinicians using diagnostic algorithms and peak flow meter measurements at the EPILAB sites. In Tanzania, the research team selected patients in collaboration with health workers in the catchment area health facility with the aim of ensuring balanced representation by gender, socioeconomic status and severity of symptoms. In Tanzania, clients with presumed CRD who had been investigated for TB, were found to be negative but remained ill and continued to visit the health facility for treatment without a definitive CRD diagnosis, were purposively selected from catchment areas of study health facilities.

For FGDs, community representatives were selected to reflect the range of literacy levels and socioeconomic backgrounds within the community in both countries. In Sudan, an additional group of adolescent girls was constituted to create a socially safe and trusting environment for open discussion, based on the research team observations that young women and adolescent girls were particularly likely to experience negative social impact of asthma in the community.

\section{Data collection}

We used common data collection methods across the two countries. Topic guides were used to guide semi-structured interviews and explored patients' and community understanding of CRD, impact of CRD on productivity, work life, finances, coping mechanisms, experiences with CRD and priorities for care. Additionally, in Sudan, patients' perceptions and experiences with the asthma management services were also explored.

Training of research assistants already experienced in qualitative research was conducted in each study site and covered overall aims of the study, data quality, ethical issues in health facility research, informed consent, privacy and confidentiality. Training included hands-on practical 
sessions on qualitative interviewing, adapting topic guides and a data collection pilot in the community. The pilot data was analysed and used to inform data collection in the main study.

Interviews were audio recorded and conducted in the local language (Swahili in Tanzania and Arabic in Sudan) in a quiet, private and secure room within the health facility premises. FGDs were conducted in mutually agreed venues in the communities. A note taker took notes during the interviews and a debrief session was observed after interviews to reconcile any inconsistencies. All interviews were transcribed by the field team, quality checked and translated into English by professional translators prior to data analysis.

\section{Data analysis}

We used the Framework Method(20) for the management and analysis of data as it facilitates transparent analysis and allows teams of researchers to collaborate on analysis(21). To improve trustworthiness, members of the research teams independently read and re-read manuscripts, inductively identifying emerging codes and developed an analytical coding framework for coding the rest of the data after reviewing a few transcripts together and resolving discrepancies. This process was iteratively repeated till no new codes emerged. Separate analytical frameworks were developed for patient interviews and focus group discussions. Codes were organised into categories and themes for analysis by charting into a spreadsheet matrix. Separate coding frameworks were developed for each country. Similar themes that emerged from the interviews were compared and discussed to identify final common themes. Socioeconomic impacts were defined as those effects of CRS that negatively influenced social life and means of livelihood of the patients with CRS and their families in the community, including the psychological consequences of these impacts(22).

\section{Results}

\section{Demographics of study participants}

We conducted 23 in-depth interviews, 7 in Tanzania (4 males, 3 females) and 5 in Sudan (2 males, 3 females). The composition of the FGDs is shown in table 1 below. There were 128 FGD participants, 50 in Sudan and 78 in Tanzania, with each FGD group consisting of 7-12 participants. Youth participants were defined as those aged less than 18 years. The FGD location and age, gender, and number of FGD participants is shown in table 1. 
Table 1: FGD location and characteristics of FGD participants

\begin{tabular}{|l|l|l|l|}
\hline FGD location & Age group & Gender & Number of participants \\
\hline Sudan & & & \\
Al-Musalmia & $>30$ & Male & 7 \\
& $>30$ & Female & 7 \\
Masoudia & $15-17$ & Female & 10 \\
Rihanna & $15-29$ & Male & 9 \\
Sarasirr & $>30$ & Male & 9 \\
& $>30$ & Male & 8 \\
\hline Tanzania & & & \\
Mvumi & $18-30$ & Male & 9 \\
& $18-30$ & Female & 10 \\
Chamwino & $>30$ & Male & 9 \\
& $>30$ & Female & 11 \\
Mpwayungu & $18-30$ & Male & 8 \\
Haneti & $18-30$ & Female & 10 \\
Dabalo & $>30$ & Male & 9 \\
\hline Total & $>30$ & Female & 12 \\
\hline
\end{tabular}

161

In general, participants described how CRDs impacted their activities of daily living, relationships and interactions within the community, and the implications of this on their overall wellbeing. The results are presented in the following themes: limitations on livelihoods; economic impacts of healthcare seeking costs and CRD mortality on patients and their families; social exclusion, discrimination and stigma; and impacts on social wellbeing, mental health, and sexuality.

\section{Limitations on livelihoods}

People with CRD in both countries reported a significantly diminished capacity to do hard physical work. The impact on livelihoods was the same among males and females as both relied largely on physical work. This resulted in both direct and indirect economic impact for people with CRD and their families due to lost working hours and inability to continue with physical work. For most people this was due to being unable to be as 'productive' in farming or informal work, but for some this involved reduced working hours in formal employment. Many people with CRD had to stop work to attend clinics, be hospitalised, recuperate after treatment and/or attend follow-up appointments. While some people with CRD could change their work to involve less vigorous and less physically demanding tasks, others stopped working entirely.

"I have been affected terribly, especially in my daily responsibilities. Our job, especially we Tanzanians, most of our activities are heavy duties, most of the activities are performed in the sun, 
most of them are in dusty working conditions on the land. I find it difficult, I can't work effectively" (IDI, Male, Patient, Tanzania).

Subsistence farming was common in our study sites with any surplus produce being sold for cash'. Therefore, reduced ability or inability to work impacts on both the family's food supply and their cash income. Loss of income also meant inability to pay school fees for dependent children and inability to take care of dependents, including aged or infirm older relatives. A female cleaner working in a local primary school reported that she stopped working when she developed CRD because of the associated fatigue and breathlessness:

"In the past, I used to do any work and move around. Since I developed this lung problem, I stopped" (IDI, Female, Patient, Sudan).

A male patient lost working days to illness and reported a negative impact on employer perceptions of his ability: even when he made himself available to do some work:

"Honestly, this problem has affected me a lot because I can't engage in activities to earn my income since I fear for the problem I am having. When you go to the sites looking for a job, you may miss for some days because of your health problem, someone looks at you and says; 'This one can't do this job'. Therefore, I am being affected in many ways such as taking care of myself and my family financially." (IDI, male CRD patient, Tanzania).

\section{The economic impact of healthcare seeking and mortality from CRD}

Many people with CRD reported several costs incurred while seeking healthcare, including expenditures on transportation to the health facility and procurement of diagnostic tests and treatments.

A teacher with asthma in Sudan reported huge transportation costs resulting from long distance travel to access specialist treatment: "And even if I want to go to Khartoum or Madani to see a specialist, I'd need to pay 1000 SDG [2.4 USD] for transportation only, and that's almost my salary, let alone the doctor's fee and medications and tests" (IDI-Male, Patient, Sudan). In both countries, transportation costs were especially high when the health facility was located far away from the patient's community and this challenge was so significant that some community members suggested that they would be willing to pay specialist consultation fees if the specialist could come to a health facility nearer to them, obviating the need for transportation. In some cases, people with CRD were reportedly accompanied on their multiple health facility visits and admissions by bread-winning family members who also incur transportation costs and loss of working hours during these periods. 
"The family will also be affected; they (relatives) will accompany the patient when s/he falls ill. All livelihood activities will be put on hold whether these activities are in the private or public work...The whole family will all be at hospital. Financially and physically, they will be exhausted." (FGD-FemaleSudan)

Participants described multiple visits to seek care in different health facilities. This journey would usually start with the local pharmacy and may end up in high level health facilities often located far away from the person with CRD. This involved not only transportation costs but also costs of medications and tests which are often beyond the ability of the person with CRD to afford . A participant mentioned that treatment could cost an entire month's salary:

"I'm dependent on my salary, but medications are very expensive and so is the doctor's appointment, in addition to the original expenses of life in general. Some way or another it'll affect you because the cheapest medicine is worth a month's salary." (IDI, Female, Patient, Sudan).

The death of a relative with CRD was also reported to impact the economy of the extended family in Tanzania. A participant reported that, in the event of death of a patient, relatives would be expected to take over their responsibilities such as care of the family, parenting, payment of school fees, provision of basic needs and management of assets, all of which exert major economic pressures on the patient's relatives.

"I had a relative who had such a problem, we came to realize when it was too late, and she had a family. When she felt sick, she used to go to the hospital to perform some tests and get medication. In the end, they realised that her lungs were badly damaged. Unfortunately, she died, and we, as relatives, have started helping and supporting the kids left behind for their education. So, it affects the family" (FGD, female adult, Tanzania).

\section{Social exclusion, discrimination, and stigma}

Many participants described a sense of exclusion from community social life related to their inability or reduced ability to participate in routine personal and community activities such as going to work, attending social gatherings, and doing household chores and cooking. A participant in Sudan reflected on limitations on her social activities after being discharged from hospital following an exacerbation of asthma: "You see now after the two months I spent at the hospital, whenever I go to an occasion I'll only participate in the conversation, I can't help and serve as I used to. I used to help with cooking the food and washing the dishes, but now even the conversation might start a coughing seizure for me" (IDI-Female, Patient, Sudan). Similarly, in Tanzania, a male patient expressed frustration at his inability to participate in the digging of grave, an important activity for men when 
there is a death in the community. "I may participate in community activities by just being there and see what is going on, but I can't fully participate because, for instance, there might be a funeral, I attend the funeral, but I can't participate in digging a grave." (IDI, Male, Patient, -Tanzania).

Stigma was described by participants in both countries as a major social impact of CRD and resulted in part from association of chronic cough with infectiousness. Stigma was both enacted (actions of discrimination by others) and internalised (withdrawal by affected individual because of their own negative feelings or anticipation of others' negative responses) and resulted in isolation from peers and the wider community.

In Tanzania, some people with CRD described how they excluded themselves from community gatherings such as the market or church, as chronic cough is mostly associated with TB and HIV in the community and raised concerns about transmitting infections among members of the community.

"I used to go to church, but when I went there, I was coughing a lot, and everybody used to look around to see who was coughing frequently? I decided not to go [anymore]. Since [then], I have never gone to church" (IDI, Female, Tanzania).

Participants commonly spoke of chronic cough as infectious and mentioned that people with chronic cough were stigmatised because they were presumed to have TB or HIV. A male participant in Tanzania highlighted this:

"Stigmatization exists here to a great extent because coughing is also a symptom of HIV/AIDS." (FGDMale-Mvumi-Tanzania). Stigmatization here referred to people moving away from anyone coughing openly and frequently for a prolonged period.

Community members also reported avoiding people with CRD for fear of getting infected. For example, some male community participants referred to a scenario where people with CRD would be avoided by potential sexual partners due to fear of contracting HIV:

"If someone is coughing frequently, people would suspect him to have acquired HIV and they will fear him think he is HIV-positive, he will no longer participate in other issues. Even some girls would be fearing him and would not want to have sex with him" (FGD, male youth, Tanzania).

Similarly, in Sudan, asthma was believed to be infectious and people with CRD may be labelled as 'TB' because of chronic cough: 
"For them, asthma is something infectious that needs to be treated or a disease that might be possible to cure. For instance, they tend to say that this person (is) living with tuberculosis or something else." (IDI-Female-Sudan). 'Them' here refers to members of the community.

Some people with CRD who internalised their stigma mentioned that they did not disclose their illness to family members and the community. In Sudan, some people with CRD reported that they pretended to be well when in the company of other people, hiding their illness from family and friends. They explained that this was to avoid attracting attention to themselves which may further reinforce stigma, increasing their sense of themselves as a burden to their neighbours or family. A young male with asthma highlighted how important it was to him for the family to be protected from feeling his 'pain':

"Yes it does...it hurts me a lot, but I don't want my family to feel my pain." (IDI-Male, Sudan)

Of particular importance to female community participants in Sudan was the potential for loss of marriage opportunities due to being discriminated against by potential suitors when a young woman developed CRD or tuberculosis. This is because both CRDs (such as asthma) and tuberculosis are stigmatising illnesses in the community . Marriage is a very important part of the identity of a woman in Sudanese society and a symbol of her status both of her family and her own position within it. A female participant who spoke of the impact of CRD on young unmarried women, described the difficulties a young woman or girl with CRD would have in attracting a suitor:

"It will lead to single marital status. It will lower the market value." (FDG, Female adult, Sudan). The impact of CRD-related stigma on marriage was reported to not only be limited to a prospective wife but also to her family. This was reported as having the potential to jeopardise future marital prospects for other women in the family:

"It is a social problem, solely a social problem. [Even] if the girl is so beautiful like the moon, they will tell you not to marry from her family as they have so and so." (FGD, Female, Sudan) .

Similarly, a male participant emphasised that prospective husbands may be discouraged from marrying young women with asthma for fear that asthma may be passed on to the children:

"In another area, people start to avoid and say that person is contagious, this is a social part. They say $X$ shouldn't marry $Y$ because she has asthma and may affect children after that, pulling them to those diseases" (FGD, Male, Sudan). 


\section{Impact on psychosocial wellbeing}

Participants reported that inability to play the usual expected roles in the society had impacts on their sense of belonging and contributions to the society. This represents an important intersection between the economic, social and psychological impacts of CRD on the patient and their families. In both contexts, the social impacts of CRD such as impaired community participation and restrictions on social activity, led to psychological stress to both people with CRD and their families. People with CRD used terminologies such as 'moody', 'angry', and 'crying' to describe the impact of CRD on their psyche. A patient in Sudan described himself as always in a bad mood and angry:

"I am always in a bad mood, and I feel exhausted to the point that I am not able to leave my bed. Very angry." (IDI, Male, Sudan)

For others, crying was a frequent means of communicating feelings at home when overwhelmed with emotions because of CRD.

"Sometimes we communicate only in tears. I feel like I can't talk and just want to cry "(IDI, Female, Sudan)

The sense of uncertainty around the illness was also reported to be shared by the entire family: "if there is someone sick in the house, the whole of the family will be in stressful condition particularly if the patient could not breathe ... The whole family will be anxious; not only the patient but all the family members will be in stressful condition." (FGD, Female, Sudan)

The impact of CRD symptoms on sexual activity was also reported to be a contributor towards negative impacts on wellbeing. Several male community members in Sudan emphasised that CRD symptoms could impact the desire and capacity for sexual activity, and this was gendered, as it was an important indicator of wellbeing and pride among men in the community.

"To get to the bottom of this issue, if I have the bad disease, I will be psychologically depressed. I have (something) in my head that I am going to die. I will not have the desire to sleep with my wife, reproduce or have children." (FGD-Male-participant-Sudan). The use of the term 'bad disease' here highlights the participant's perception that chronic cough was 'bad' in line with the general perception that chronic cough was due to tuberculosis or HIV which would invariably end in death. 
332 We found multiple socioeconomic impacts of chronic respiratory disease on people diagnosed or suspected of having the disease, on their families and communities. Both CRD symptoms and treatment seeking resulted in a significant economic toll on people with CRD and their families because of impairment of physical fitness and ability to work, loss of working hours and jobs, and direct and indirect costs of health seeking such as costs of transportation to the health facility and treatment and opportunity costs of missed work. Our findings further show that stigma associated with chronic cough resulted in social exclusion. The social impacts including inability to carry out activities of daily living, impaired community participation, non-disclosure to families and restrictions on sexual activity in the context of stigma and social exclusion, were found to be a source of significant psychological stress on people with CRD and their families.

The strong association of chronic cough with TB in these settings is not surprising. Chronic cough was widely presumed to be TB in a similar study among Ugandan communities(23). This highlights the huge importance of TB as an ongoing public health challenge in the sub-Saharan African context. Whilst Tanzania is one of the 30 high TB burden countries, Sudan has a moderate but also significant burden of TB (WHO Global TB report 2020). The common knowledge of TB in the communities may be explained by the historicity of endemic TB disease and by the substantial investments into TB control compared to CRD and other diseases in these contexts. A downside of this association, as seen in this study, is the stigmatization and ostracism of people with CRD in the community. This may lead to failure to attend health facilities for fear of being confirmed as having TB disease, which further delays diagnosis and can lead to increased morbidity and mortality $(13,24)$. Misinformation about chronic cough is another driver of stigma seen in this study. A study investigating knowledge and perceptions of asthma among secondary school students in Tanzania revealed that most information about asthma had been passed on to students by their parents and non- asthmatic students presumed that asthma was infectious and therefore avoided interactions with their asthmatic colleagues for fear of contracting the disease (10). Systematic community sensitization activities with trained and well-informed health personnel could help improve community perception about chronic cough and contribute to tackling stigma in CRDs. Clear messages about the causes of asthma and the availability and effects of treatment on controlling symptoms would help to destigmatise asthma and other CRDs. The effect to seeing people being managed well with affordable medicines and transform their lives can be more powerful than being told about treatment effectiveness. Words also matter and consistent use of terms like 'people who have asthma' rather than asthma 'victims' or CRD 'sufferers' can start to shift mindsets. There are many transferable lessons to be learned from community de-stigmatisation efforts with HIV in the 
antiretroviral therapy era(25) and, more recently, efforts being applied to COVID-19 related stigma(26).

The economic impact of CRD in these communities highlights the vulnerability of people with CRD in mostly rural settings, who are self-employed or working in informal sectors of the economy such as subsistence farming, and without recourse to any social security. Chronic diseases, including CRDs, are known to incur significant economic costs for both patients and the health system $(27,28)$. In Malawi, the mean care seeking cost for chronic cough per patient was found to be 2.3 times the average cost per capita on health for the country and consisted mainly of transport and drug costs(29). Similarly, TB-related catastrophic costs have been well recognised and documented. A systematic review of financial impact of TB in LMICs revealed that on average the total cost was equivalent to $58 \%$ of reported annual individual and $39 \%$ of reported annual household income(30). To tackle catastrophic costs in TB context, poverty reduction strategies are increasingly being woven into TB control programmes including social protection initiatives such as cash transfers, food baskets and social insurance (31). Health systems could draw from these experiences and interventions in TB to develop an integrated approach to addressing the economic impacts of CRDs in the sub-Saharan African setting.

Transportation costs were strongly highlighted by our study participants as a major source of economic loss, impacting people with CRD and relatives who accompany them on hospital appointments. Our informants themselves made the that the transportation costs were often the equivalent of their monthly salary. Transportation has been identified as an important social determinant of health (32) and a well-documented barrier to engaging in the care of chronic diseases especially among poor, vulnerable populations (33). Studies done in high income settings have shown that interventions such as provision of bus passes, taxi vouchers and reimbursements from insurance covers could improve healthcare utilization (34) but these interventions may not be feasible in our study setting . because of lack of transport infrastructure. Policy makers in our study context could consider decentralisation of services and investment in specialist mobile clinics to rural areas and geographically distant communities as measures to reduce the impact of transportation costs and improve health outcomes of people with CRD. In our study setting, the extended family system and the social interconnectedness meant that any economic costs incurred by the person with CRD could lead to a ripple effect within their social circle, potentially setting up or exacerbating vicious cycles of poverty and ill-health. Life course studies have established that risk factors in childhood and adolescence, including poverty, contribute to subsequent development of CRD in adulthood (35). The economic impacts observed in this study risk perpetuating poverty and worsening outcomes such as morbidity and mortality. Measures to mitigate these impacts would be 
crucial in breaking the cycle of poverty and ill-health, improving outcomes in subsequent generations. Developing a clear diagnosis and care pathway for CRD in our study setting would be an important part of this mitigation.

Failure to engage in usual activities of daily living, disruptions in sexual activities and difficulties with community participation reported in this study have also been shown to compound several chronic diseases including asthma(36), COPD (37) and HIV/AIDS (38) Physical challenges of managing the disease can sometimes affect mood and emotional health leading to anxiety and depression in both people with CRD and the carers (39). Health system responses to CRD should include supporting carers and families by telling them what to expect, encouraging them to present to care at the earliest recognition of symptoms and patient-centred communication to support with selfmanagement of symptoms where appropriate. Research in other contexts shows that recognising and managing anxiety and depression can increase ability to stick with prescribed COPD treatment, improve physical health and reduce medical costs(40).

The finding in this study that young Sudanese women with chronic cough and their families were discriminated against by potential suitors further highlights the gendered nature of stigma associated with chronic cough. The perception that asthma, a major cause of chronic cough well known in this society, was primarily hereditary, has also been described in a previous study of asthma in urban Sudan (41), where $67 \%$ of 490 asthmatic patients believed that asthma could be transmitted within the family. The study also highlighted denial of asthma diagnosis and non-use of inhalers by young female asthmatic patients as a coping mechanism to avoid stigma. This highlights the disproportionate social burden of CRD born by young women. A related finding in our study is the choice made by people with CRD to hide their symptoms from family and community members in a bid to keep the emotional burden of illness away from them. Young people with chronic illness have been shown to be particularly circumspect about disclosure of their illness, frequently choosing non-disclosure because of perceived fear of rejection, pity, and perceptions of being seen as vulnerable or different(42). In the context of stigma, non-disclosure could also be a coping mechanism to avoid stigmatization while shielding the family from its effects, as observed in our study.

\section{Limitations of the study}

As this was a qualitative study, quantitative costs were not collected, and quality of life not measured. Quantification of economic impact through a cost of illness study would lay the foundation for a future evaluation of the cost-effectiveness of interventions to address CRD within the health systems in these settings. However, the direct testimony of the people with CRD and 
members of their communities about their lived experience, provides important information about the range of social, economic and psychological impacts that should be measured in our study setting and insight into potential interventions to address them. Whilst the findings are not statistically generalisable to populations in Sudan, Tanzania or sub-Saharan Africa more widely, the common issues arising within these two different contexts are likely to be generalisable to similar settings, whilst the context-specific issues point to the importance of locally developed interventions.

\section{Conclusion}

440 Chronic respiratory diseases are associated with significant social and economic impacts among people with CRD and their families in Sudan and Tanzania. While the impacts on livelihood and economy resulted mainly from impairment of physical ability to carry out income-generating activities and from expensive healthcare costs, the long-standing association of chronic cough with TB and HIV/AIDs in our study settings was a major driver of stigma and social exclusion experienced by people with CRD and their families. This highlights the negative central role of these chronic stigmatizing diseases in framing perceptions and attitudes towards other illnesses in the community. Context-appropriate social safety nets and systematic community health education and sensitization would be required to address the economic and social impacts of CRD identified in this study, as well as the broad causes and management of chronic cough in the communities. In addition, provision of available, accessible and affordable care for CRD is necessary to break the cycles of poverty, ill-health and stigma in these sub-Saharan African contexts

\section{Declarations}

\section{Ethical considerations}

The study was approved by the ethics committees of National Institute for Medical Research, Tanzania (ref /Vol.IX/2922); the Ministry of Health of Gezira state, Sudan (ref 44/T/KH/1) and the Liverpool School of Tropical Medicine (ref 18-043). Permission was also sought from the respective district authorities. Written informed consent was obtained from all participants. Additional consent for participation in the adolescent FGD group was provided by parents of the girls. We ensured confidentiality of the IDIs by conducting interviews in secure locations away from third parties where the patients were comfortable and by not capturing names of the patients. Additionally, patients were assured that the discussion would not be shared with any third parties and the identifying characteristics such as occupation and town were securely locked away. 
The datasets used and/or analysed during the current study are available from the corresponding author on reasonable request.

\section{Competing interests}

468 The authors declare that they have no competing interests.

\section{$469 \quad$ Funding}

470 This research was funded by the National Institute for Health Research (NIHR) (project reference $47116 / 136 / 35$ ) using UK aid from the UK Government to support global health research. The views 472 expressed in this publication are those of the authors and not necessarily those of the NIHR or the 473 UK Department of Health and Social Care.

474 TW is supported by grants from the UK Wellcome Trust (209075/Z/17/Z) and the Medical Research

475 Council, Foreign Commonwealth and Development Office, and Joint Global Health Trials 476 (MR/V004832/1).

\section{Author Contributions} $M T, R T, N N, S M, A E, E S$, and UE conceptualised and designed the study. ES, SM, RA and EH collected all data with the help of research assistants in country. UE, ES, NN, SM, EH, MT and RT analysed the data. UE prepared the first draft of the manuscript and coordinated reviews and comments on subsequent versions. UE, ES, MC, JA, TW, RA, AO, MT and RT discussed and interpreted results of the study. All authors reviewed versions of the manuscript and approved the final version for submission.

\section{Acknowledgements}

This work was a collaborative work by the International Multidisciplinary Program to Address Lung Health and Tuberculosis in Africa (IMPALA) Consortium. We thank the IMPALA management team for their support. We are grateful to research assistants and colleagues from the National Institute for Medical Research (NIMR) Tanzania, National Leprosy and Tuberculosis Program (NLTP) Tanzania, Kibong'oto Infectious Disease Hospital, Tanzania and the Epidemiological Laboratory (EPILAB), Khartoum Tanzania for their role in the organisation and conduct of the study. Our gratitude also goes to the Ministry of Health, Community development, Gender, Elderly and Children (MoHCDEC),

492 Tanzania; Dodoma Regional and Chamwino District government authorities, and the Gezira State 493 ministry of Health, Al Gezira, Sudan for their support. Finally, we are grateful to the people with CRD, their families and communities who participated in this study. 
496 Emmanuel Addo-Yobo, Brian Allwood, Hastings Banda, Imelda Bates, Amsalu Binegdie, Adegoke

497 Falade, Jahangir Khan, Maia Lesosky, Bertrand Mbatchou, Hellen Meme, Kevin Mortimer, Beatrice

498 Mutayoba, Louis Niessen, Jamie Rylance, William Worodria, Heather Zar, Eliya Zulu, Jeremiah

499 Chakaya, Lindsay Zurba and S Bertel Squire.

500

501

502

503

504

505

506

507

508

509

510

511

512

513

514

515

516

517

518

519

520 
522 1. Collaborators GBDCRD. Prevalence and attributable health burden of chronic respiratory diseases, 1990-2017: a systematic analysis for the Global Burden of Disease Study 2017. Lancet Respir Med. 2020;8(6):585-96.

2. WHO. Globl surveillance, prevention and control of chronic respiratory diseases. A comprehensive approach. Geneva: World Health Organization; 2007.

3. Kurmi OP, Semple S, Simkhada P, Smith WC, Ayres JG. COPD and chronic bronchitis risk of indoor air pollution from solid fuel: a systematic review and meta-analysis. Thorax. 2010;65(3):2218.

4. Osman RK, Mortimer K, Bjune G, El Sony Al. Chronic respiratory disease in adults treated for tuberculosis in Khartoum, Sudan. Public Health Action. 2016;6(3):199-204.

5. Egere U, Shayo E, Ntinginya N, Osman R, Noory B, Mpagama S, et al. Management of chronic lung diseases in Sudan and Tanzania: how ready are the country health systems? BMC Health Serv Res. 2021;21(1):734.

6. Adeloye D, Basquill C, Papana A, Chan KY, Rudan I, Campbell H. An estimate of the prevalence of COPD in Africa: a systematic analysis. COPD. 2015;12(1):71-81.

7. Buist AS, McBurnie MA, Vollmer WM, Gillespie S, Burney P, Mannino DM, et al. International variation in the prevalence of COPD (the BOLD Study): a population-based prevalence study. Lancet. 2007;370(9589):741-50.

8. Ait-Khaled N, Odhiambo J, Pearce N, Adjoh KS, Maesano IA, Benhabyles B, et al. Prevalence of symptoms of asthma, rhinitis and eczema in 13- to 14-year-old children in Africa: the International Study of Asthma and Allergies in Childhood Phase III. Allergy. 2007;62(3):247-58.

9. Ahmed R. the prevalence and risk factors of noncommunicable chronic lung diseases in adults in rural and urban Sudan Liverpool, UK: Liverpool School of Tropical Medicine; 2018.

10. Shimwela M, Mwita JC, Mwandri M, Rwegerera GM, Mashalla Y, Mugusi F. Asthma prevalence, knowledge, and perceptions among secondary school pupils in rural and urban coastal districts in Tanzania. BMC Public Health. 2014;14:387.

11. Bukhman G, Mocumbi AO, Atun R, Becker AE, Bhutta Z, Binagwaho A, et al. The Lancet NCDI Poverty Commission: bridging a gap in universal health coverage for the poorest billion. Lancet. 2020;396(10256):991-1044.

12. Rajeswari R, Balasubramanian R, Muniyandi M, Geetharamani S, Thresa X, Venkatesan $P$. Socio-economic impact of tuberculosis on patients and family in India. The international journal of tuberculosis and lung disease. 1999;3(10):869-77.

13. Courtwright A, Turner AN. Tuberculosis and stigmatization: pathways and interventions. Public Health Rep. 2010;125 Suppl 4:34-42.

14. Tanimura T, Jaramillo E, Weil D, Raviglione M, Lönnroth $K$. Financial burden for tuberculosis patients in low-and middle-income countries: a systematic review. European Respiratory Journal. 2014;43(6):1763-75.

15. Brakema EA, Tabyshova A, van der Kleij R, Sooronbaev T, Lionis C, Anastasaki M, et al. The socioeconomic burden of chronic lung disease in low-resource settings across the globe - an observational FRESH AIR study. Respir Res. 2019;20(1):291.

16. Hammarberg K, Kirkman M, de Lacey S. Qualitative research methods: when to use them and how to judge them. Hum Reprod. 2016;31(3):498-501.

17. Smith JA, Osborn M. Interpretative phenomenological analysis as a useful methodology for research on the lived experience of pain. Br J Pain. 2015;9(1):41-2.

18. El Sony Al, Chiang CY, Malik E, Hassanain SA, Hussien H, Khamis AH, et al. Standard case management of asthma in Sudan: a pilot project. Public Health Action. 2013;3(3):247-52.

19. Tongco MDC. Purposive sampling as a tool for informant selection. Ethnobotany Research and applications. 2007;5:147-58. 
20. Ritchie J, Lewis J, Nicholls CM, Ormston R. Qualitative research practice: A guide for social science students and researchers: sage; 2013.

21. Gale NK, Heath G, Cameron E, Rashid S, Redwood S. Using the framework method for the analysis of qualitative data in multi-disciplinary health research. BMC medical research methodology. 2013;13(1):1-8.

22. Darin-Mattsson A, Fors S, Kareholt I. Different indicators of socioeconomic status and their relative importance as determinants of health in old age. Int J Equity Health. 2017;16(1):173. 23. van Gemert F, Chavannes N, Nabadda N, Luzige S, Kirenga B, Eggermont C, et al. Impact of chronic respiratory symptoms in a rural area of sub-Saharan Africa: an in-depth qualitative study in the Masindi district of Uganda. Prim Care Respir J. 2013;22(3):300-5.

24. Pungrassami P, Kipp AM, Stewart PW, Chongsuvivatwong V, Strauss RP, Van Rie A. Tuberculosis and AIDS stigma among patients who delay seeking care for tuberculosis symptoms. Int J Tuberc Lung Dis. 2010;14(2):181-7.

25. Camlin CS, Charlebois ED, Getahun M, Akatukwasa C, Atwine F, Itiakorit H, et al. Pathways for reduction of HIV-related stigma: a model derived from longitudinal qualitative research in Kenya and Uganda. J Int AIDS Soc. 2020;23(12):e25647.

26. Organization WH. Social Stigma associated with COVID-19. World Health Organization; 2020. 27. van Boven JF, Vegter S, van der Molen T, Postma MJ. COPD in the working age population: the economic impact on both patients and government. COPD. 2013;10(6):629-39.

28. Sapkota T, Houkes I, Bosma H. Vicious cycle of chronic disease and poverty: a qualitative study in present day Nepal. Int Health. 2021;13(1):30-8.

29. Sichali JM, Khan JAK, Gama EM, Banda HT, Namakhoma I, Bongololo G, et al. Direct costs of illness of patients with chronic cough in rural Malawi-Experiences from Dowa and Ntchisi districts. PLoS One. 2019;14(12):e0225712.

30. Tanimura T, Jaramillo E, Weil D, Raviglione M, Lonnroth K. Financial burden for tuberculosis patients in low- and middle-income countries: a systematic review. Eur Respir J. 2014;43(6):1763-75. 31. Patel BH, Jeyashree K, Chinnakali P, Vijayageetha M, Mehta KG, Modi B, et al. Cash transfer scheme for people with tuberculosis treated by the National TB Programme in Western India: a mixed methods study. BMJ Open. 2019;9(12):e033158.

32. Ruggiano N, Shtompel N, Whiteman K, Sias K. Influences of Transportation on Health Decision-Making and Self-Management Behaviors among Older Adults with Chronic Conditions. Behav Med. 2017;43(1):61-70.

33. Starbird LE, DiMaina C, Sun CA, Han HR. A Systematic Review of Interventions to Minimize Transportation Barriers Among People with Chronic Diseases. J Community Health. 2019;44(2):40011.

34. Tierney WM, Harris LE, Gaskins DL, Zhou XH, Eckert GJ, Bates AS, et al. Restricting medicaid payments for transportation: effects on inner-city patients' health care. Am J Med Sci. 2000;319(5):326-33.

35. Rylance S, Masekela R, Banda NPK, Mortimer K. Determinants of lung health across the life course in sub-Saharan Africa. Int J Tuberc Lung Dis. 2020;24(9):892-901.

36. ANDREW N, Booth T. The social impact of asthma. Family Practice. 1991;8(1):37-41. 37. Eisner MD, Blanc PD, Yelin EH, Sidney S, Katz PP, Ackerson L, et al. COPD as a systemic disease: impact on physical functional limitations. The American journal of medicine. 2008;121(9):789-96.

38. Taraphdar P, Guha RT, Haldar D, Chatterjee A, Dasgupta A, Saha B, et al. Socioeconomic consequences of HIV/AIDS in the family system. Niger Med J. 2011;52(4):250-3.

39. Jácome C, Figueiredo D, Gabriel R, Cruz J, Marques A. Predicting anxiety and depression among family carers of people with chronic obstructive pulmonary disease. International psychogeriatrics. 2014;26(7):1191. 
619 40. Smith SM, Sonego S, Ketcheson L, Larson JL. A review of the effectiveness of psychological 620 interventions used for anxiety and depression in chronic obstructive pulmonary disease. BMJ open 621 respiratory research. 2014;1(1):e000042.

622 41. Merghani T, Zaki A, Ahmed A, Toum I. Knowledge, attitude and behaviour of asthmatic 623 patients regarding asthma in urban areas in Khartoum State,Sudan. Khartoum Medical Journal. 624 2011;04(1):524 - 31.

625 42. Kaushansky D, Cox J, Dodson C, McNeeley M, Kumar S, Iverson E. Living a secret: disclosure 626 among adolescents and young adults with chronic illnesses. Chronic illness. 2017;13(1):49-61. 